\title{
AN EFFICIENT CONTRIBUTION TO COMPUTING THE SKYLINE ON GPU
}

\author{
Hadjer Belaicha ${ }^{1 *}$, Lougmiri Zekri ${ }^{2 *}$ and Larbi Sakhri ${ }^{3}$ \\ ${ }^{1,2,3}$ Computer Sciences Department, Ahmed Ben Bella Oran1 University, \\ BP 1524, El-M'naouer, Maraval, \\ Oran, Algeria \\ ${ }^{1}$ hadjer-belaicha@hotmail.fr, ${ }^{2}$ lougmiri@yahoo.fr, ${ }^{3}$ larbi.sekhri@univ-oran.dz
}

\begin{abstract}
The skyline computation is very important in the field of decision making. It gives solutions to help among a wide dataset and where information is contradictory, especially when the implemented solution is progressive. As the need to get rapid solution is growing, it will be suitable to exploit new machines' performances and plate-forms. In this paper, we present a new solution of type divide-and-conquer for computing the skyline on GPU (Graphics Processing Units) cards. The proposed partitioning is adaptable to characteristics of the GPU. This proposition can lead to a well balanced computing and avoids overflows. The dominance tests are performed on points components in parallel and dominated points are early discarded unlike other solutions which save them for next loops. This way of comparison avoids threads' idleness. We compare our solution with other similar solutions on the same datasets. Experimentations show that our proposition is better in terms of time computing and exploitation of the GPU parallelism.
\end{abstract}

Keywords - skyline, GPU, multi-criteria, optimization, GNL, GSA, threads, partitioning

\section{INTRODUCTION}

In a lot of situations, returned responses by search engines are not convincing, this is due to the adversarial features of data. For example, in an industrial firm it is hard to find a compromise between the maximization of the production and the reducing of $\mathrm{CO}_{2}$ emission. Really, a lot of parameters have to be respected for resolving such situations. The deduction of the well parameters is not easy; so, a tool for decision making can be so helpful for taking the best choices.

In this context, the calculation of the skyline of a set of points has become an important paradigm in the field of decision making research. Skyline computation helps users to choose the best from enormous amount of available data by identifying a set of interesting responses (called also objects). For example, a hotel classification system is queried to find hotels that are cheap and that are close to the sea; while, hotels which are close to the sea are more expensive than those far away. Thus, it is difficult to bring out the best hotel that meets both criteria. The skyline operator consists of locating the set of competing objects of a certain database according to the dominance relation, noted " $>$. In this paper, a tuple of a database $S$ is called also an object. In terms of spatial geometry, this tuple is represented by a point $p$ which belongs to some vector space $E$. The numeric

Received: March 21, 2019

Reviewed: May 8, 2019

Accepted: June 12, 2019

* Corresponding Authors 
attributes of $S$ form the dimensions of $E$. In terms of multi-objective optimization, the attributes form what is so-called the criteria. We use interchangeably the terms point, object and tuple for meaning the same thing. Also, the term dimension designates the term criteria.

The dominance relation is defined as follows: Supposing that $D$ is the set of $d$ criteria $D=\{1,2, \ldots, d\}$. Consider two objects $p$ and $q$ of the database $S$. For $1 \leq i, j \leq d, p$ dominates $q$ noted $p>q$, if and only if the next condition is verified:

$$
p>q \Leftrightarrow\left(\left(\forall d_{i} \in D / p\left(d_{i}\right) \leq q\left(d_{i}\right)\right) \wedge\left(\exists d_{j} \in D / p\left(d_{j}\right)<q\left(d_{j}\right)\right)\right)
$$

When $p \ngtr q$ and $q \ngtr p$, then $p$ and $q$ are said to be non-dominated or competing [24]. From the data set $S$, the skyline operator returns the set of points that are not dominated by any other, in all dimensions $D$ [2]: $S k y_{\mathrm{D}}(S)=\{p \in S \mid \nexists q \in S: q>p\}$

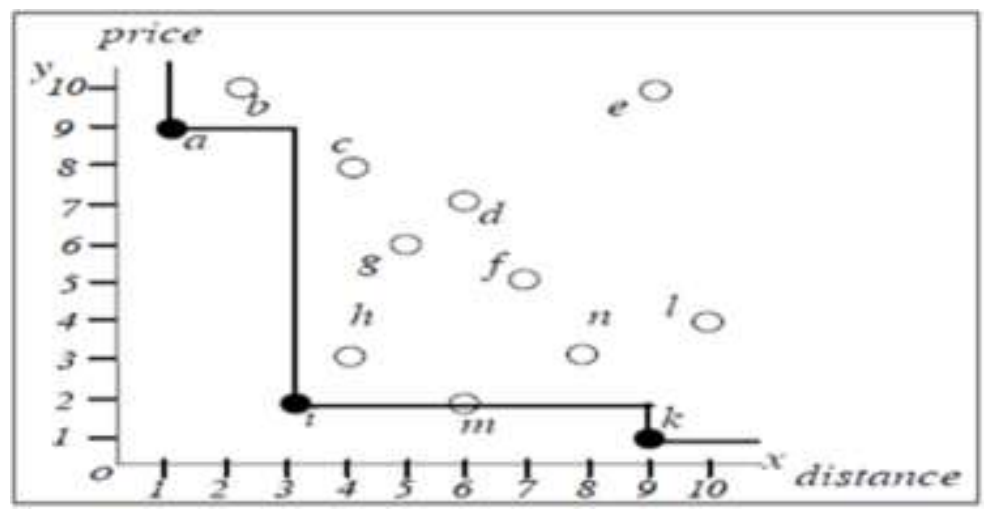

Fig. 1 Example the skyline of hotels database [16]

According to this definition and by considering the example depicted in Fig. 1 [16], the hotels $a$ and $k$ have the minimal values on the axes distance and price respectively, therefore, no other point can dominate them, thus $a$ and $k$ form a part of the skyline. The hotel $i$ can not be dominated by any other point, it has neither the minimal distance to the beach nor the minimal price. The skyline operator will return $\{a, i, k\}$ as the set of responses.

By its definition, the dominance relation is irreflexive, anti-symmetric and, especially, transitive. Any algorithm which deals with the skyline computing must be careful with these dominance properties. We will show later, that the execution of some algorithms has been slow as they did not pay attention to these properties.

Historically, the dominance relation was intensively worked in mathematics and economics since Vilfredo Pareto has given his theory about "Indifference curves". In the multi-objective optimization and operational research fields, researchers have developed ingenious methods for determining the so called "Pareto front". In 1975, [11] has studied extensively the problem of finding all maximal elements of a set of vectors. In 2001, the term skyline was firstly used due to the graphical representation of the Pareto front, and was defined in the database context [2]. Since this date, several works have responded to this problem in different systems such as centralized single-and many processors (CPU) systems and distributed multiprocessor (multi-CPU) systems. Other works have been implemented on new plate-forms like MapReduce. The objective is to treat more and more amounts of data, since current databases have become wider. But few works have exploit machines with graphical processors, we have encountered only three works of two research teams, more details will be given later.

In order to treat huge amounts of data, companies, such as NVIDIA and AMD, have developed new GPU cards for allowing the development and the execution of general codes as a calculation tool for Powerful High Performance. These graphic processors were initially used for 3D gaming and multimedia applications. Actually, these 
capabilities are exploited more broadly to accelerate computational workload in multiple domains. Graphics processors have become true platforms of intensive computing and allow lightening the CPU of a certain amount of work. They draw their attraction, in solving problems of scientific computation, from two important points. First, the GPUs are of a massively parallel architecture. It remains for users to find the efficient ways to exploit the GPUs. This means that GPU will allow a significant acceleration of the calculation codes. Secondly, the low cost of graphics cards is of significant economic interest compared to the price of a conventional microprocessor. These two points explain the active use of GPUs.

In this paper, we present a solution of type BNL(block Nested Loop)[2] which opens several windows in parallel and it is of type DC (Divide-and-Conquer) [2] as we propose a partitioning according to the properties of the considered GPU card. Thus, our partitioning is adaptive. The proposed solution does not use any preprocessing; it manipulates the data in its raw form. Our solution exploits the maximum parallelism offered by the graphics card. The idea behind of our proposition avoids the processes idleness of which could suffer the works turning on this platform. This paper is an extension of our paper [1] which has appeared in 2016. Historically, our solution is the third one which exploits the GPU card, as we will show in related works section. We present more explanations, details and executions than in [1].

The remainder of this paper is as follows: The second section gives a state of the art of calculating Skyline since its birth. GPU architecture seen by CUDA is presented in Section 3. Section 4 presents a review of GNL. In Section 5, details about the partitioning problem are given. Section 6 presents our proposition. In Section 7 describe our experimental results. Finally we conclude in Section 8.

\section{RELATED WORK}

The skyline operator and his calculation have attracted a lot of attention since 2001 with the appearance of [2]. This work has adapted the calculation of the Pareto front in the context of databases. This is mainly due to the importance of skyline results in many applications, such as, multi-criteria decision making, data mining, visualization and user preferences expressed by a given query.

Since that date, several approaches have been proposed to make the calculation more efficient. In the context of our paper, we classify these approaches according to an architectural aspect. In another words, we classify them according to the system architecture on which every approach works. [24] gives a classification according to the behavior of approaches; this behavior is defined by the progressivity property [10]. An algorithm is said to be progressive if it returns the correct skyline points during its execution, like NN [10], while a non-progressive one returns these points after it terminates its execution like DC [2]. These approaches can be divided into two main classes. The first one brings together all the solutions that run in the CPU. This class, itself, is divided into three subclasses. The first subclass is formed by the set of algorithms which run on centralized CPU. The second subclass groups the algorithms which run on distributed CPU while the algorithms of the third subclass run on multi-core architecture.

Among the algorithms that run on centralized CPU, we can find Block Nested Loop BNL algorithm [2]. This algorithm applies directly the domination test. It defines a window with some size $t$ and inserts $t$ concurrent points as a current skyline. At each time, BNL reads a new point $p$ and compares it with the current skyline. If $p$ is dominated then it will be discarded else dominated points by $p$ are deleted from the window and $p$ will be inserted. If at a certain moment $p$ is concurrent with the current skyline then an additional temporary file is used for storing $p$. At the end of the execution, BNL calls the temporary file for comparing its points with the current skyline for a final decision. For a 
not large number $n$ of points, BNL executes $O(n)$ operations. This is considered as the best execution case if a skyline point is already put in the window. The Divide and Conquer DC algorithm [2] divides the input data set into several partitions according to the median on every dimension. Exactly, $2^{d}$ partitions, where $d$ is the number of dimensions, will be obtained. At a first time, the partial skyline of each partition is calculated. At a second time, DC merges all these partial skylines to find the final one. DC proposes also to apply a multi-way partitioning.

The paper [19] has proposed two progressive algorithms for computing the skyline queries. The first one is called Bitmap. In the first step of this algorithm, authors construct bitmap vectors (indexes) for representing points according to distinct values on every axis. In the second step, authors compare these vectors bit by bit [24]. This comparison leads to a rapid detection of the skyline. The most disadvantage of this method is that the high cost for constructing these indexes. [19] presents also the algorithm Index. For a ddimensional space, Index creates and sorts $d$ lists; after, it compares the points in a circular manner for collecting the point's coordinates. Index is an efficient progressive algorithm; it avoids the test of huge parts of lists.

The Nearest Neighbor NN algorithm [10] uses R-Trees as an index structure. NN finds the nearest point $p$ to the origin $O$, by considering the Euclidean distance, and then divides the space along each axis with respect to that point. If we are with a 2 dimensional space, the projection on the two axes will generate a rectangle where $p$ and $O$ are its main heads. The points which do not belong to the rectangle will be eliminated. The processes of searching the nearest point to the origin and projection will be repeated more times, till discovering the skyline points. If we are in front of a 3-dimensional space, the projection will generate a hypercube. $\mathrm{NN}$ is well suited for progressive applications. It works efficiently in a two-dimensional space, but suffers from problems of duplication during eliminations when the dimension exceeds four [24]. Branch and Bound Skyline BBS algorithm [16] computes nested spaces [24] and executes NN recursively. It terminates when the corresponding nearest neighbor query does not retrieve any point within the corresponding space. BBS uses R-trees; consequently it suffers from the same NN's problems like the high number of nested spaces to be computed. In order to remedy the problem of CPU occupation, [7] has coupled BNL and presorting for giving SFS (Sorted First Skyline). Experimentations have shown that this combination reduces memories accesses and CPU-occupation. The authors of [23] have proposed a new structure which they called Skycube. This structure consists in calculating all possible combinations of the lattice in order to generate the skyline according to their two (Bottom-Up Skycube) and (Top-Down Skycube) algorithms.

In the second subfamily, several algorithms have been designed for distributed applications. For example, [12] is an algorithm that computes the skyline in a distributed environment using views. These ones store a pre-calculated skyline result of a subspace of the input. In order to exploit new platforms, [14] has presented the algorithm MapReduce Grid Partitioning based Multiple Reducer Skyline Computation MR-GPMRS. This study presents the effects of parallelization on the skyline computing on different datasets by varying the dimensionality, the cardinality and the execution buffers. MR-GPMRS was compared with the algorithms published in [25]. [21] presents a framework on MapReduce for computing independent regions after generating the convex hull of a query. The final skyline is the union of all skyline regions.

In multi-core architectures, the cores involved in a processor and communicate simply by updating the main memory. In order to exploit this architecture, the authors in [13] published APSkyline for the skyline computation in a multi-core system. It proposes the angle-based partitioning model [20]; then, APskyline adopts the paradigm partition-execute-merge framework where the dataset is split into $\mathrm{N}$ 
partitions (one for each core). The local skyline set for each partition is computed, and finally the skyline set is determined by merging these local skyline sets.

The second class groups the rest of works which solve the skyline on the GPU. Few studies have been proposed in this class. [6] is the first published work which has computed the skyline by using SIMD model on GPU. The main objective is to exploit the high parallelism offered by the GPU device. Authors propose to correct the naïve nested loop method and extend it to apply GNL for GPU-based Nested Loop. We must see here that GNL is not an extension of BNL [2]. Authors have compared their GNL to BNL, which is executed on CPU, for showing that experimentally their method outperforms BNL. One main purpose of [6] is to minimize the number of dead threads during execution. This idleness is caused by the unbalanced workload in a thread block [6], like we will show later in this paper. GNL operates mainly in two phases. In the first one, it divides the database into subsets on different blocks where each thread tests a point with all other points in the same block. In the second phase, the algorithm merges the partial skylines of the different blocks in the same way. This simple method can be used as a naive method applicable on GPU, but the lack of communication between threads during execution causes unnecessary tests that increase execution time. More details about GNL will be given later.

[3] presents a GGS (GPGPU skyline) algorithm that aims to share the load between the CPU and the GPU in a way to take full advantage of their capabilities. Authors use the CPU to keep track of the data to be processed on the GPU, as well as filtering points by eliminating those points that are dominated, while the GPU handles batches of data of fixed size $\alpha$ where $\alpha$ denotes the number of points to be compared with a single point at each iteration. The main idea of this algorithm is to sort the input according to a monotone function; in turn, each thread compares each point of the data set with the first $\alpha$ points of the same set, until the entire database is processed; although, this algorithm is suitable for GPGPU applications. In this algorithm, the estimation of the parameter $\alpha$ depends on the type of input data. In 2015, authors of [4] have presented SkyAlign which is a new algorithm for computing the Skyline on GPU. At the difference of GGS [3], SkyAlign proposes to make a static partitioning scheme different to the dynamic one presented in [3]. This static partitioning allows a better control of the branching divergence in order to exploit the transitive relationships between points. SkyAlign gives priority to the work efficiency and respects the through-put at the expense of a maximal through-put. By this proposition, we think that authors try to avoid problems due to eventual overflows. It seems that authors correct the set of problems caused by the instability of CUDA programs, especially those caused by the threads concurrency.

Due to the large lattice which can be obtained when we speak about SkyCube and materialized views [17], [23], and in order to exploit the multithreading capabilities of new machines (CPU and GPU), Authors present three intensive parallel procedures in [5]. They give three intensive parallel procedures for computing the skycube. In this context, the dataset is distributed horizontally on cores and every core computes the skyline of its partition. The major aim of this work is to construct a general framework which works on both CPUs and GPUs.

$\mathrm{Yu}$ Boseon in [22] presents HashSkyline and HashSkylineGPU for computing the skyline. As pre-processing, a monotonic hash function was defined. It permits to map objects to cells for accelerating accesses to them in some defined hash tables. More of this, a pivot combined to a correlation threshold is also computed for representing sets of objects. But surprisingly, HashSkylineGPU was not compared to any solution which turns on GPU; it seems like a theoretical aspect.

As we have said before, globally, there are two sets of algorithms which compute the skyline [24]. The first set of algorithms does not use any processing on the input; hence, the contained algorithms are applied directly on the raw data. It results that there is only one time of computation. The second set of algorithms is applied on preprocessed data. 
Consequently, there are two times for consideration: preprocessing time and computing time.

In this work, we have chosen to compare our proposition to this one published in [6] as this later does not use any preprocessing like us for computing the skyline on GPU.

\section{GPU ARCHITECTURE SEEN FROM CUDA}

Based on the stream concept, [8] gave four classes for classifying computer architectures. The concept behind stipulates that it is possible to do a classification according to the number of instruction steams and data streams. In the first class, we can find the SISD (for Single Instruction and Single Data) architectures. This classical Von Neumann architecture means that in this type of architectures, only one instruction is executed on one data. So, it is about a sequential computer which does not present any degree of parallelism. The second class contains MISD, for Multiple Instructions Single Data, architectures. In this class, computers do a pipelined execution where different instructions, like "Read data by a processor-Decode Data by next processor-Display data by a third processor,.." and so on. The interesting third class is formed by SIMD computers where SIMD means that a Single Instruction is applied in parallel, on different processors, on Multiple, so different, Data. The last class is formed by MIMD, for Multiple Instructions Multiple Data, architectures. In this case, different computers execute different instructions or programs. In this paper, we are concerned by SIMD architectures as it is very interesting and is largely applied on vector computers.

Graphics hardware is actually an interesting platform for executing intensive applications which demand high performance capabilities in programming and resources. These graphics hardware offer a high number of processors which can be executed in parallel for speed up execution.

NVIDIA Corporation is a world provider of graphics processors [15] and is faithful to SIMD processors class. Coupled to CUDA, it is possible to write C programs for the purpose of high computing and exploiting graphics cards. Actually, other languages like Java or Python can be used for exploiting GPU.

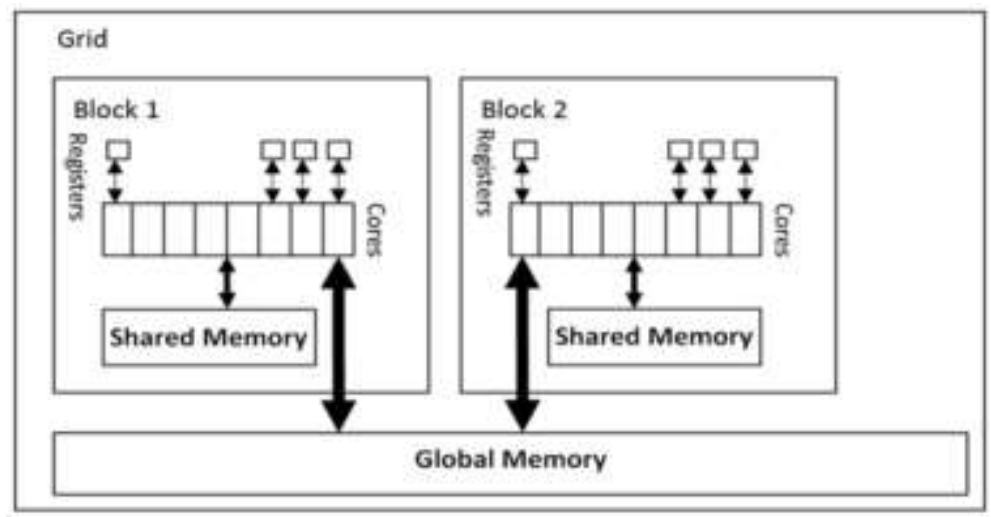

Fig. 2 A simplified GPU Architecture

A GPU (for Graphics Processing Unit) is a grid which contains, essentially, a global memory and blocks of small independent SIMD multiprocessors. The cores, or threads, according to CUDA, of every block run concurrently and access to a shared memory. This shared memory is visible only in the block and is faster than the global memory. More of this, a set of higher speed registers is implemented in every block. These registers are used for storing temporarily intermediate results. Fig. 2 presents a reduced schema for GPU. This GPU description implies that more the global memory is avoided more the programs are faster. The communication between CPU and GPU is provided by a slow medium. It is recommended to avoid multiple transfers between them. In our 
work, we try to divide and charge data on the shared memory according to the hardware card properties for a better exploitation of the machine.

\section{REVIEW OF GNL}

GNL [6] is the first work that has proposed to use graphics hardware for computing the skyline queries. This work tried to improve the nested loop algorithm for this purpose. More of this, GNL took charge of reducing the idleness of threading during the process of computing the skyline. In the literature of the nested loop algorithm [18], the program sweeps all different combinations of the manipulated sets, like addition of two matrices. In some and not useful cases, a certain redundancy is done. This one can produce an extra-time which can cause a delay in computing softly a problem.

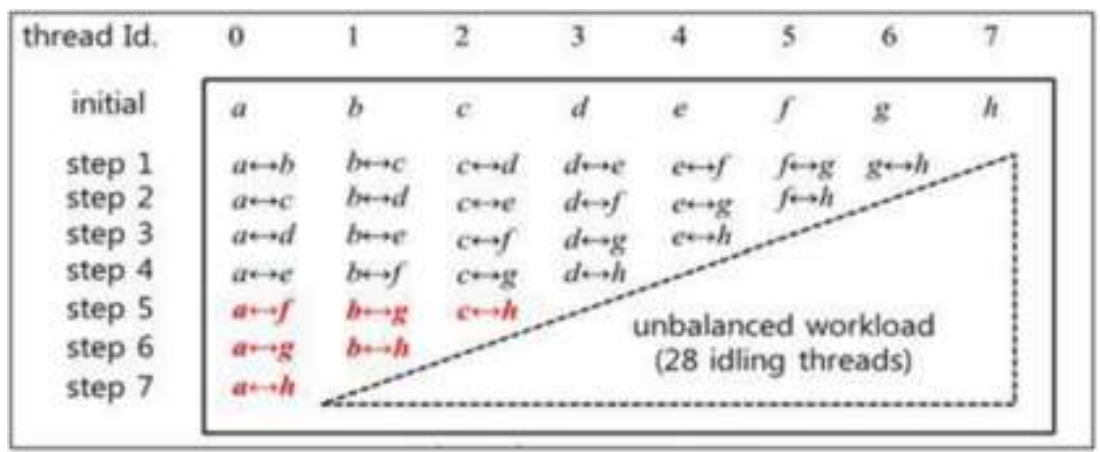

Fig. 3 The naive method for computing the skyline [6]

In [6], authors gave a naive form for computing the skyline in one block, as depicted in Fig. 3 [6]. For the 8 points $a, b, c, .$. and $h, 8$ threads are designated, one thread by point, to perform the dominance tests between these points. The comparison process is done in 7 steps. Fig. 3 shows how many threads are un-useful or idle, more of this some threads are note executed, like thread 7 , or less used like thread 6 . At the opposite, some threads were highly executed, like thread 0 . In total, an unbalanced workload results from this naive nested loop. If we consider a higher number of blocks in CUDA, a large amount of idle threads is produced which leads to a waste of the effort.

\begin{tabular}{|c|llllllll|}
\hline thread Id. & 0 & 1 & 2 & 3 & 4 & 5 & 6 & 7 \\
initial & $a$ & $b$ & $c$ & $d$ & $e$ & $f$ & $g$ & $h$ \\
step 1 & $a \leftrightarrow b$ & $b \leftrightarrow c$ & $c \leftrightarrow d$ & $d \leftrightarrow e$ & $e \leftrightarrow f$ & $f \leftrightarrow g$ & $g \leftrightarrow h$ & $h \leftrightarrow \rightarrow a$ \\
step 2 & $a \leftrightarrow \leftrightarrow c$ & $b \leftrightarrow d$ & $c \leftrightarrow e$ & $d \leftrightarrow f$ & $e \leftrightarrow g$ & $f \leftrightarrow h$ & $g \leftrightarrow a$ & $h \leftrightarrow b$ \\
step 3 & $a \leftrightarrow \rightarrow d$ & $b \leftrightarrow e$ & $c \leftrightarrow f$ & $d \leftrightarrow g$ & $c \leftrightarrow h$ & $f \leftrightarrow \rightarrow a$ & $g \rightarrow b$ & $h \leftrightarrow c$ \\
step 4 & $a \leftrightarrow e$ & $b \leftrightarrow f$ & $c \leftrightarrow g$ & $d \leftrightarrow h$ & 4 & 4 idling threads \\
\hline
\end{tabular}

Fig. 4 The modified Nested Loop presented in GNL [6]

GNL operates into two stages for computing the skyline. In the first stage, GNL presents a modified nested loop algorithm for reducing the number of idle threads and, by the way, reducing the unbalanced workload. Fig. 4 illustrates this schema. Instead of letting threads 5, 6 and 7 idle, the dominance tests in one thread block are executed in a circular manner. These dominance tests are called IntraBlockTest. In the second stage called the InterBlockTest, the dominance tests are performed between blocks. GNL tests 
all saved objects in the memories. In order to evaluate the performance of GNL, authors have compared their work with BNL which is executed in the CPU.

In reality, we reproach to authors this proposition and the comparison mode. It is not well to compare BNL, which it is executed on one poor CPU, with GNL, which executed on thousands of graphics processors.

The important imperfection with this idea is why authors have not exploited the transitivity of the dominance relation. When an object $a$ dominates an object $b$ the dominated one must be rapidly discarded. It is clear on Fig. 4 that a lot of redundant tests are performed many times. These redundant tests will surely affect time execution and higher workload will take place.

\section{THE PARTITIONNING PROBLEM}

The first algorithms were implemented without any data preprocessing [2]. The manipulation of raw data can lead to a more time of computing the skyline while it is possible to apply a preprocessing during the time data acquisition. Historically, [19] is the first work which made a processing on data in this field of treatment. More details are given in [24] about preprocessing methods.

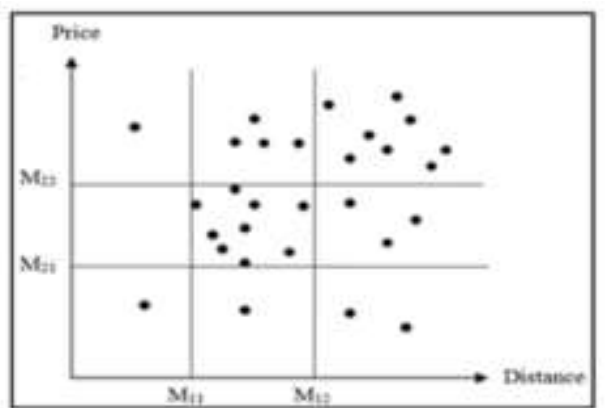

(a) Grid partitioning

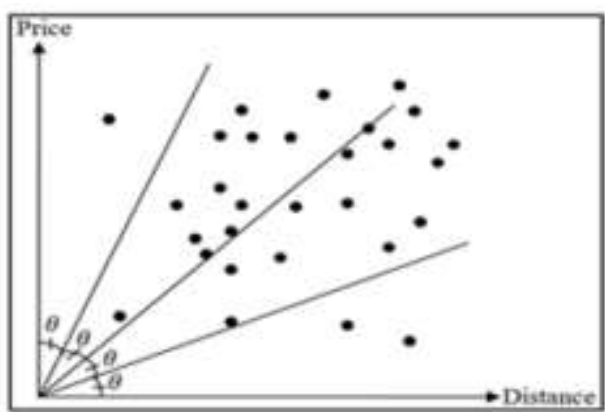

(b) Angle partitioning

Fig. 5 Two example of data partitioning

For a d-dimensional space, [2] proposes to apply two manners for partitioning data when applying the DC algorithm. In the first manner, authors suggest to divide data according to the median in a one way fashion. This proposition produces $2 \mathrm{~d}$ partitions therefore $2 \mathrm{~d}$ partial skyline must be computed and merged after. In the second manner, authors propose a multi-way fashion for decomposing the input. thus, more partial skyline must be computed. The problem of partitioning is not simple. Due to the nature of the input (see [2] and [24]), the density of points in partitions is not equal. In some partitions the number of points can be very high in some parts and very low in other parts. Consequently, the dense parts will impose higher workloads. The entrusting of partitions to blocks will generate an unbalanced workload. In an implementation point of view, a hard synchronization between block threads must be made, otherwise, rapid blocks can respond early and force the rest of blocks to terminate.

For example, Fig. 5 presents a partitioning based on grids and another one based on angles. In both schemas, we can see that the density of points is not balanced in all partitions. It is possible to make a second dynamic partitioning for balancing the density like in [13].

In our case, we have chosen to apply a partitioning according to the machine properties. This way can give a real balanced workload on all block threads and redundant tests will be avoided as we can see later in this paper. 


\section{THE GPU SKYLINE ALGORITHM GSA}

Although several algorithms have been proposed to solve the skyline but their resolution remains costly in execution time. This imperfection is due to CPU dominance tests. To reduce this cost, most skyline algorithms have thought of avoiding unnecessary dominance tests as early as possible by eliminating non-skyline points [24] or by partitioning the input data set into several partitions as in the Divide-and-Conquer algorithm [2].

The precedent works have not said anything about where the temporarily dominant points and partial skylines are saved during the skyline computing, especially as the number of points is very high and a single round of test domination is insufficient.

From this point of view, it is important to properly model an efficient algorithm on GPU, in order to:

1. Reduce the cost of the dominance tests;

2. Limit the transfer of data between CPU and GPU;

3. Exploiting the maximum resources and the capacity of the GPU.

As we have said before, it is suitable to use the shared memory more than the global one for accelerating the computing. We propose to compute the partial dominance tests on the shared memory, and merging the deposited partial skyline points on the global memory during the loading of next parts on the shared memory. In other words, we couple, in parallel, the execution on both memories. This procedure leads to a rapid execution. For the best of our knowledge, it is the first time such combination is done.

\subsection{GPU SKYLINE ALGORITHM}

In this part, we present a new method for the skyline computation on GPU. We call it GSA for GPU Skyline Algorithm. GSA uses the Single Instruction Multiple Data (SIMD) model and GPU functionality for parallel computing the dominance tests. GSA is of type divide-and-conquer algorithms and is designed to take into account the limitations of memory saturation and avoids the execution of unnecessary dominance tests unlike GNL.

Let $E$ a set of $n$ points in a vector space of $d$ dimensions. These dimensions are the numerical criteria on which the calculation will be made. The steps of GSA are as follows:

1. Collecting the card graphics properties. Let $n b$ the number of card blocks and $n t$ the number of threads in every one;

2. Dividing the input into $n b$ parts. Every part will be assigned to one block, for computing its partial skyline. This part is called BlockPart. By this way and at the difference of precedent partitioning schemas, the density of points is equal in all parts. We will assign $n b p=\left\lceil\frac{n}{n b}\right\rceil$ points to every block.

It is known that global memory GM is very large unlike SM the shared one. We must be careful during the loading of points into SM especially as temporary data will be saved into tiny registers. The rest of GSA takes place into two phases. In the first one, we calculate the partial skyline of each block. The second phase merges the already calculated partial skylines in the precedent phase to complete the final skyline computation. 


\subsubsection{PHASE 1: CALCULATION OF THE PARTIAL SKYLINE AND MEMORY MANAGEMENT}

In this phase, each block is responsible for its own data. Each BlockPart is decomposed into several blocks where $n t$ points are loaded into SM. The $n t$ threads freely access this set. This phase is presented as below:

Initially, for a reason of acceleration the computing and management of memory saturation, the initial $n b p$ points of every BlockPart is divided into $\left(\alpha=\left[\frac{n t}{d+1}\right\rceil * 2\right)$ small parts. We propose to use $d$ threads for computing dominance tests. Every thread compares two attributes of the same order of two different points. The $(d+1)^{\text {ths }}$ threads act as coordinators. Every $(d+1)^{\text {th }}$ thread decides to discard the dominated points.

Every discarded point is marked for avoiding it in next laps. Intuitively, the $d$ threads are orthogonal to $(d+1)^{\text {ths }}$ threads. Fig. 8 shows an example of such testing process. We will comment this figure later. At every lapi, we will get $\beta_{i}$ points to test with $\left(1 \leq \beta_{i} \leq \alpha\right)$ and $\left(\beta_{i} \leq \beta_{i-1}\right)$. We also propose to charge $\left(\left\lceil\frac{\beta_{\mathrm{i}}}{2}\right\rceil *(\mathrm{~d}+1)\right)$ for comparing these $\beta_{i}$ points. Therefore a necessary number of threads are called and no dominated point is tested in future laps. This means that we exploit the transitivity property of the relation of dominance. In final execution of this testing process, the partial skyline of the small block is obtained and saved in the global memory. We call it SkyParto. At every time $i$, a small part is charged and processed as we have explained above.

When a partial skyline $S k y$ Part $_{i}$ is deposited on global memory it will be merged with precedent partial skyline as illustrated in the Fig. 6. We occupy the global and shared memories in parallel. This manner of acting leads to a high and maximal parallelism for computing the skyline. For the best of our knowledge, it is the first time that such coupling is applied.

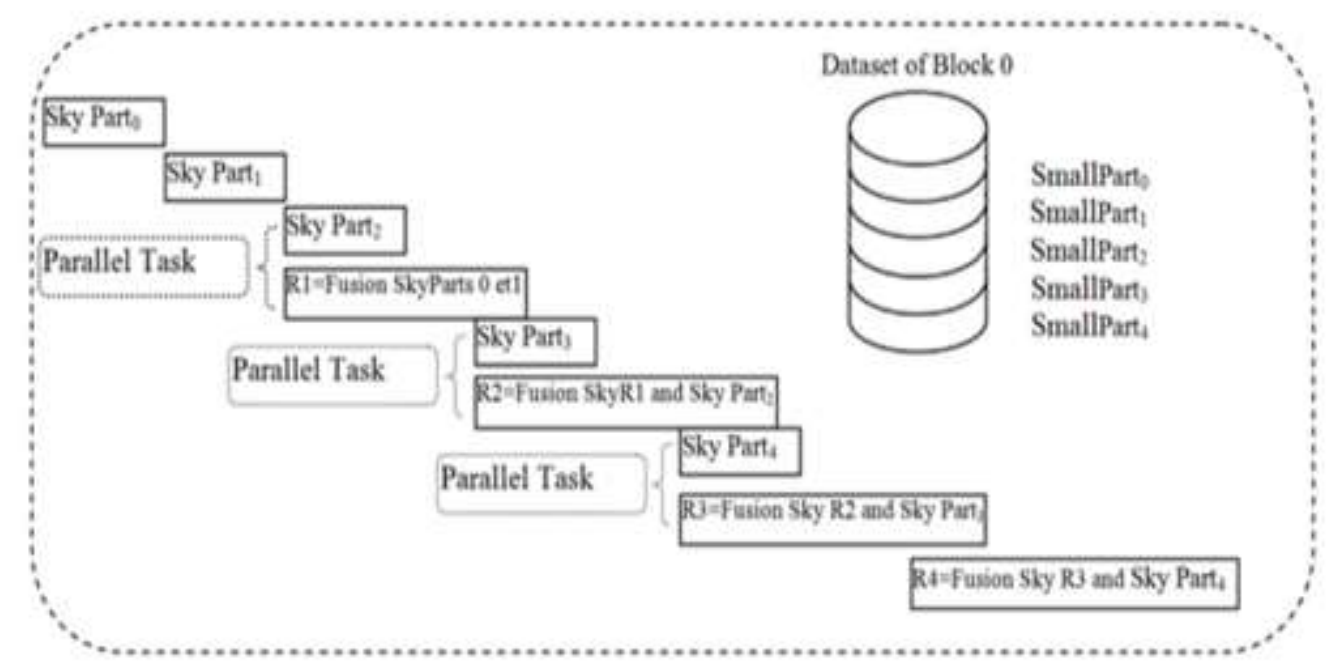

Fig. 6 Process of merging in one block

\subsubsection{PHASE 2: FINAL SKYLINE COMPUTING}

At the end of computing Phase 1, all partial skylines are deposited on the global. At this time, dominance tests between the blocks must be performed to calculate the final skyline. Since the threads of one block cannot access the data of another block, we chose to merge directly into the global memory to avoid saturation of the shared memory and to accelerate the calculation. We proceed in the Round-Robin manner that is to say that the skyline of the first block will be merged with the skyline of the second block. This result will be merged with the skyline of the next block until exhausting all partial skylines. 


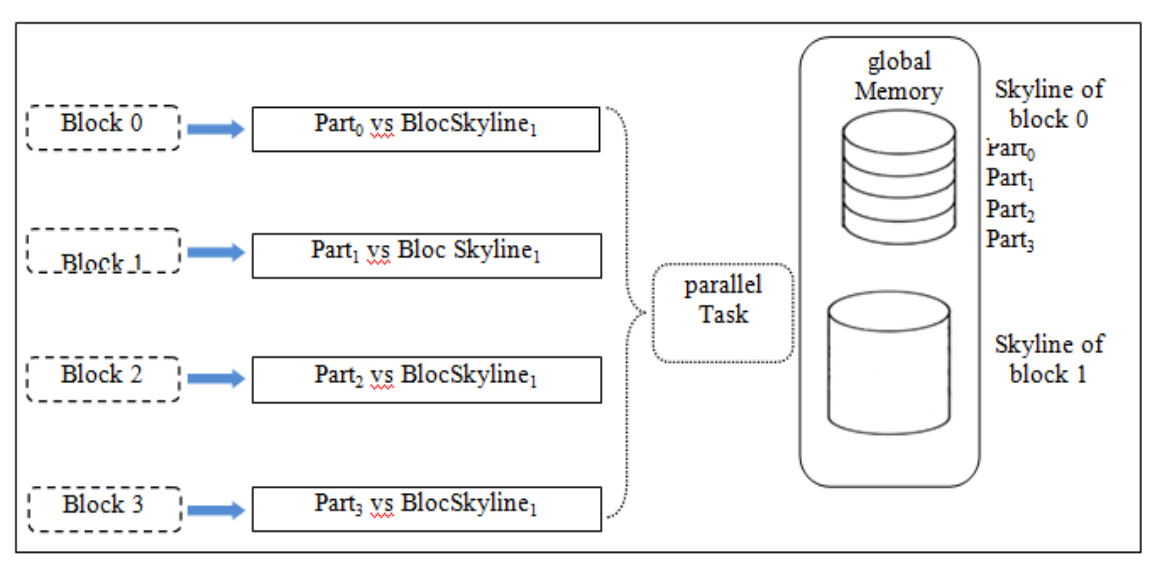

Fig. 7 Fusion of skyline blocks

To accelerate the duration of the merging between the skylines of two blocks and a maximum exploitation of the GPU, we divide the partial skyline of the first block into N parts (where $\mathrm{N}$ is the number of blocks) and each block will be responsible for the Part that it will merge with the skyline of the second block. Fig. 7 gives an illustration of the melting of the skyline of two blocks.

\subsection{MAXIMUM EXPLOITATION OF THE GPU}

Reducing execution time remains a challenge, especially when the number of criteria is important. The minimization of this time, we load $d$ threads (the number of criteria) to compare the points two by two. Each thread compares $d$ criteria. We also use a coordinator thread between the threads of a single comparison. To test the dominance between two points of $d$ criteria, we need $(d+1)$ threads (see Fig. 8), in this way the processing will be parallelized between the different threads by making maximum use of GPU resources. Table I gives an illustrative example in order to show the progress of our skyline computing. On this table, we have to compare 10 points in a 3 -dimensional space. Fig. 8 shows that we need $(3+1)$ threads for comparing two points. Every thread compares two points' attributes. The fourth thread decides which points are saved for the next round of the comparing process. For the points A and B, the coordinator deduces that they are concurrent while $D$ is discarded by the other coordinator.

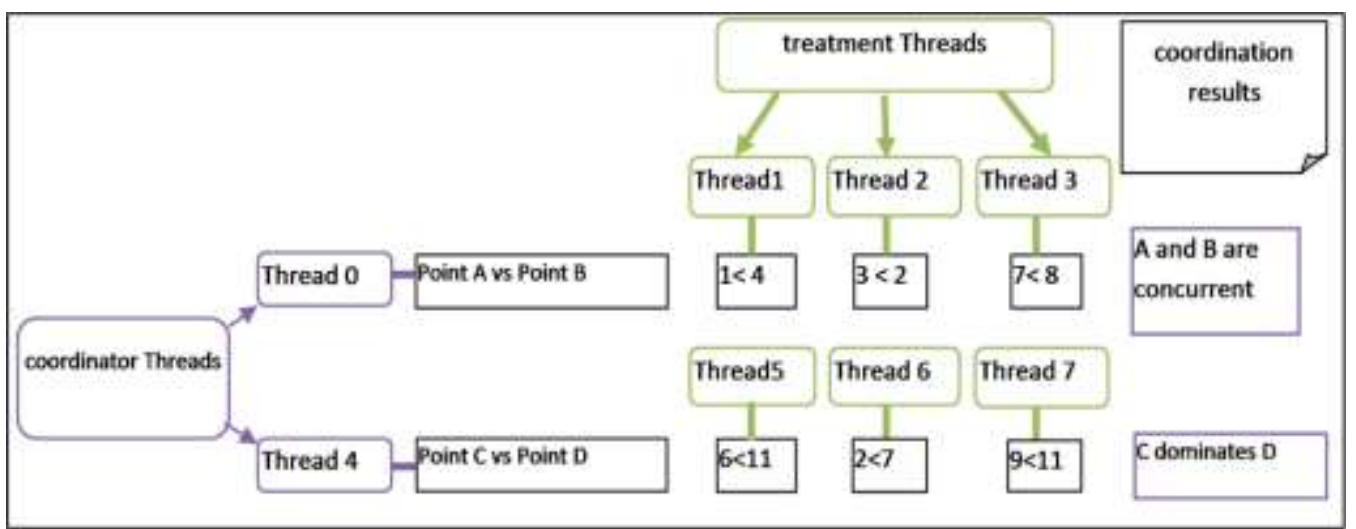

Fig. 8 An illustrative example of the comparing process in our proposition 
Table I. Example of a database with 3 dimensions

\begin{tabular}{|l|l|l|l|}
\cline { 2 - 4 } \multicolumn{1}{c|}{} & Axe 1 & Axe 2 & Axe 3 \\
\hline A & 1 & 3 & 7 \\
\hline B & 4 & 2 & 8 \\
\hline C & 6 & 2 & 9 \\
\hline D & 11 & 7 & 11 \\
\hline E & 9 & 12 & 9 \\
\hline F & 3 & 10 & 1 \\
\hline G & 7 & 6 & 1 \\
\hline H & 10 & 8 & 3 \\
\hline$Y$ & 2 & 5 & 6 \\
\hline$Z$ & 8 & 9 & 4 \\
\hline
\end{tabular}

Fig. 9 gives the diagram of sequences of GSA. The program begins by reading data from the hard disk and transfers it to the global memory. The data is charged on the shared memory after partitioning as we explained above. The $n$ threads will then compare points and deposit the partial skyline in the global memory. After the computing of the final skyline, the result is copied to the host.

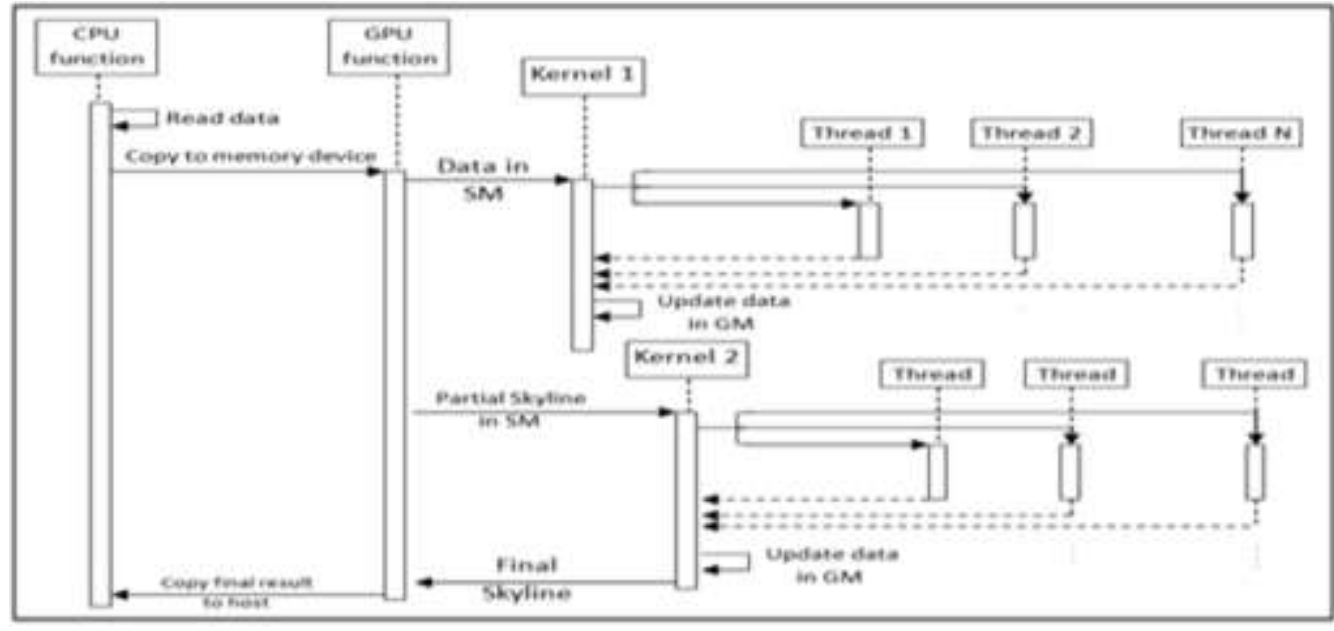

Fig. 9 Diagram of sequences of GSA

\section{EXPERIMENTATION}

The first experimentations were conducted on a machine with i7, Ram 16 Go, HDD 1 To, 08 cores/cpu and NVIDIA Quadro 6000 with 6 Go. On this machine, we have compared GSA to GNL. For the second experimentations, we have used a machine with Intel Core i5 $2.50 \mathrm{GHz}$, and a memory of $4 \mathrm{~GB}$, equipped with a graphics NVIDIA GeForce GT $525 \mathrm{M}$ with $2 \mathrm{~GB}$. On this machine, we have compared GSA to DC which was executed on CPU, like in [6] where GNL was compared BNL. All programs were written under Ubuntu 12.04. The same synthetic databases of [2] and [10] were used. These are of three data types: correlated, anti-correlated and independent.

In a first series of tests, we have compared GSA to GNL. We have written our own code for GNL as we could not get it from their owner writer.

For the first step, we have been careful for reproducing the same execution schema of GNL. We can see on Fig. 10, that the same GNL's manner of testing is implemented. We have replaced the points $a, b, c \ldots$ of Fig. 3 by $p_{0}, p_{1}, \ldots$ We can also see the same idle processes on this figure. 


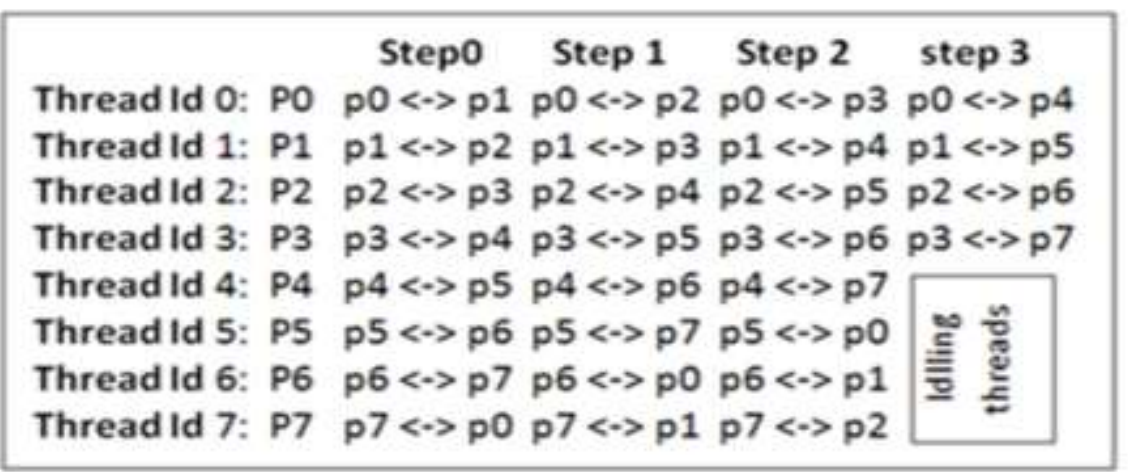

Fig. 10 The testing process in GNL

The first spectacular result is that we have found the same curves for these three synthetic dataset types [24, 2]. As a result, the variation of the type of the dataset does not imply that we will get different time consumption.

The implemented politic of partitioning and testing is behind this spectacular constant time. We have also found these same curves for GNL. This is due, also, to the constant speed of the threads. In [6], there were also constant times for the three datasets.

In the first series of experimentations on the first machine, we have fixed the cardinality to $10^{5}$ and varied the dimensionality from 5 to 30 . Fig. 11 shows that GNL has consumed less time than GNL. We can read that a nearly constant time of execution is elapsed in GSA.

We must note here, that it is about a data generated by certain probability, see [2, 24], which does not give any information about the dispersion of the points into the considered space. The augmentation of points or dimensions does not imply that the behavior of programs will change. At any time, if we introduce a fixed number of points and with one of these dimensions we will get the same time. We note here, that we charge points according to the capacity of the graphics cards, therefore we use the same parallelism degree for all points. The use of threads was also flexible and the workload is balanced between blocks as we have explained above.

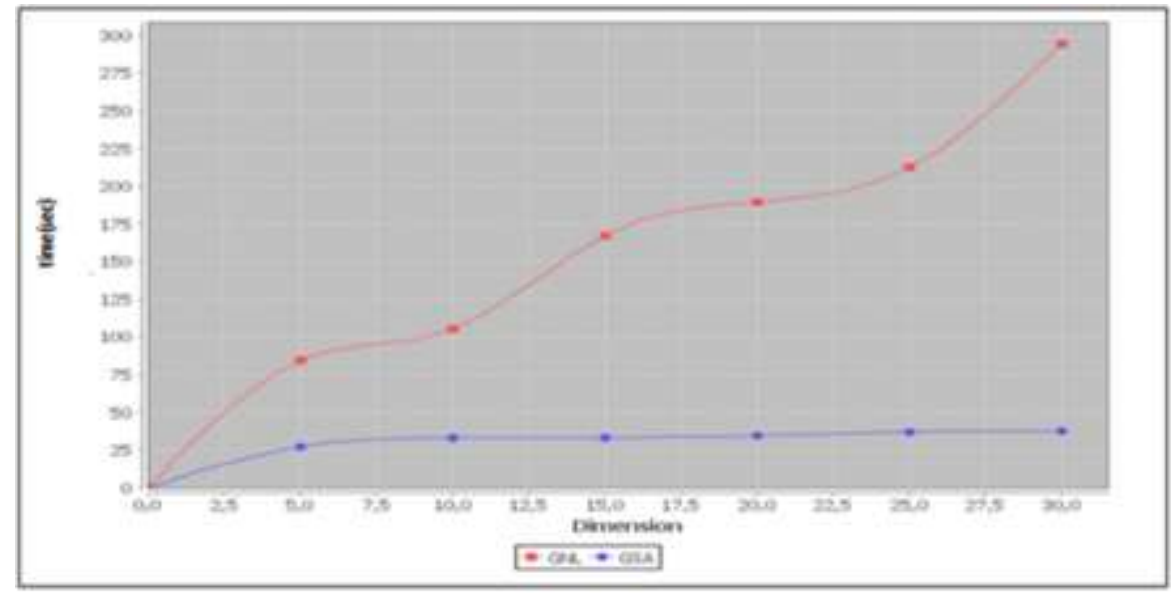

Fig. 11 Time spent by GSA and GNL when varying the dimensionality

The Fig. 11 shows clearly that the time spent in GNL increases according to the dimensionality. With no doubt, the repeated tests of dominance have their impact on GNL. The objective of GNL was to make working the threads even if some computation is repeated. If GNL has exploited the transitivity of the relation of dominance, it could take less time. 


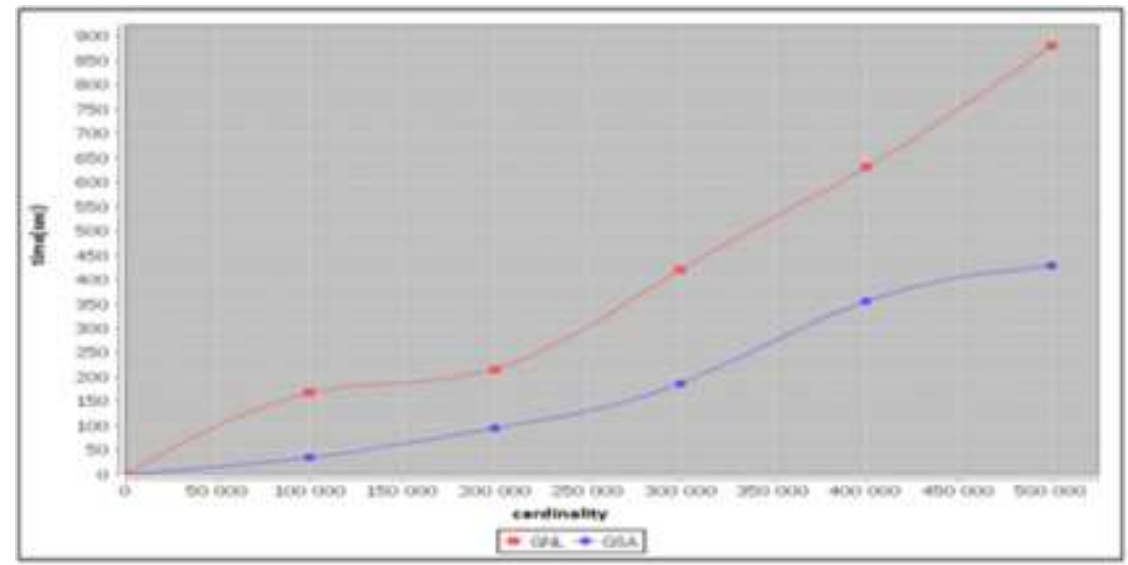

Fig. 12 Time elapsed by GSA and GNL on varying the cardinality

For the first series of experimentations on the same machine, we have fixed $d$ to 30 and varied the cardinality from 100000 to 500000 and we have counted the time consumed by GSA and GNL. Fig. 12 shows that GSA was better than GNL. It has taken less time than GNL. At the difference of the precedent experimentations, time execution increases according to the cardinality. In this case, the BlockParts are bigger comparatively to the precedent case. At the difference of GSA, GNL has taken more time for computing the skyline. We can see that the gap of time between the two algorithms increases according to the number of points injected in the system. The politic implemented in GNL penalizes it. In GNL, every thread executes more comparisons and dominated points are saved in more cycles. This strategy delays the global execution of GNL.

In the second series of experimentations on the second machine, we have injected the three synthetic datasets. For every type, we have varied the dimension $d$ from 2 to 10 and fixed the cardinality $n$ to $10^{4}$ (like in [2]) at a first time. At a second one, we have fixed $d$ to 5 and varied $n$ from $10^{4}$ to $10^{5}$ with a step equal to $10^{4}$. The objective of this experimentation is to study the difference between the execution of GSA and DC in two different hardware environments. The results of these experimentations are as follows.

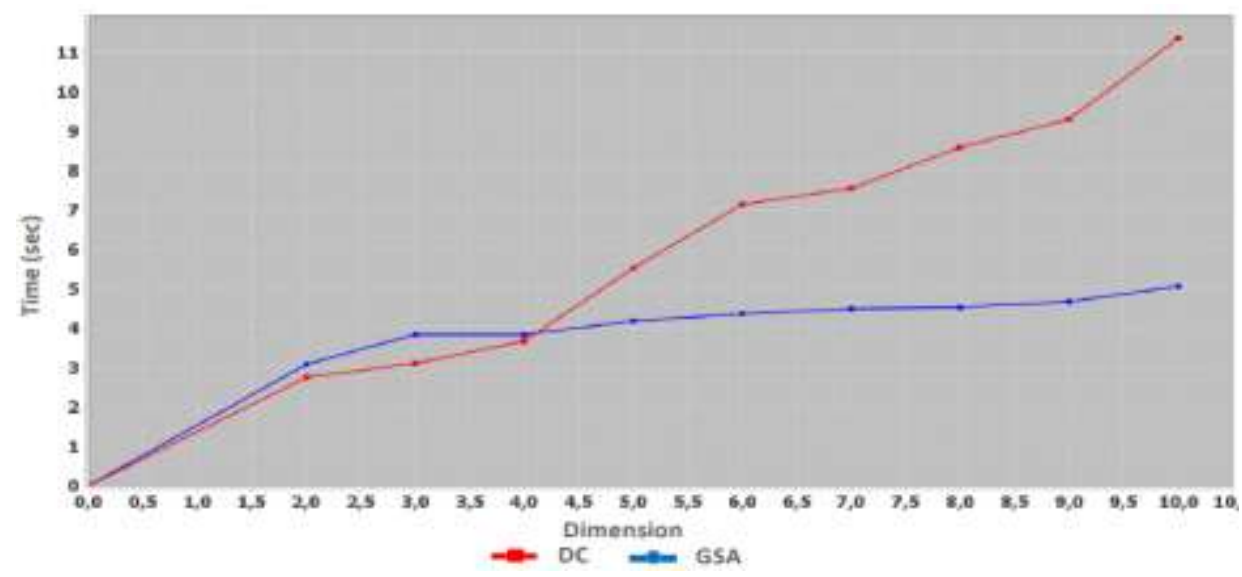

Fig. 13 (a) Elapsed time on correlated data with variation of the dimensionality 


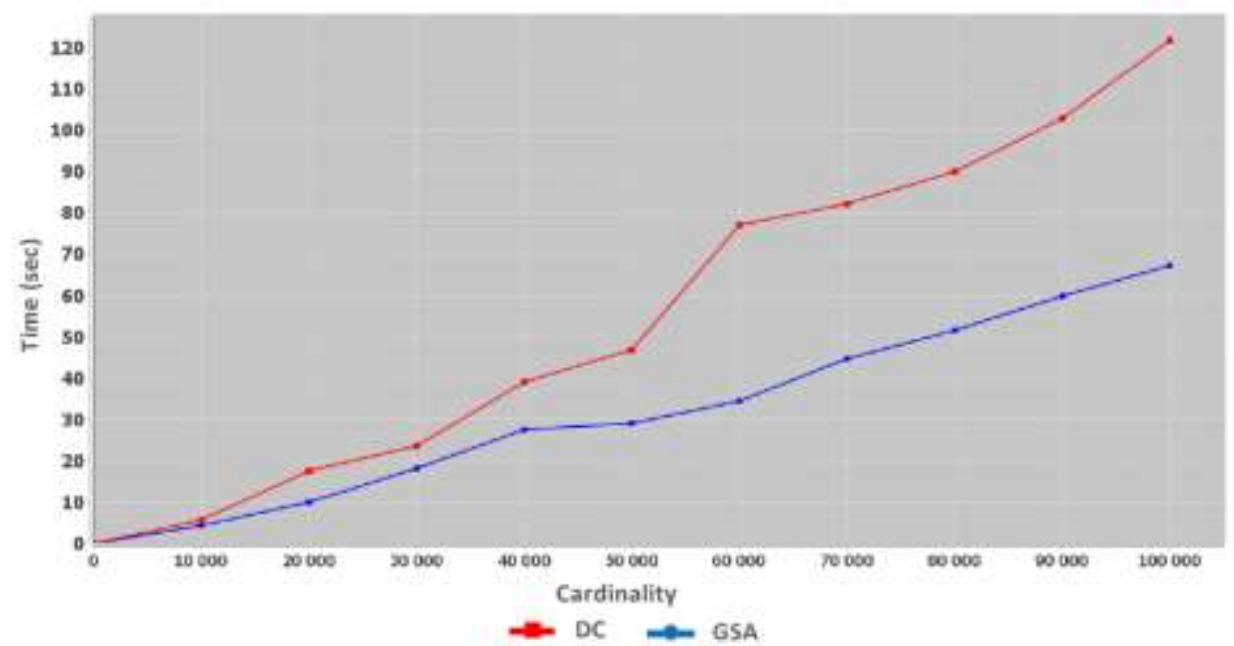

Fig. 13 (b) Elapsed time on correlated data with variation of the cardinality

Fig. 13 (a) shows that for small dimensions 2, 3 and 4, DC has been faster than GSA, but, for large datasets, GSA has taken over DC. We can see the gap between their elapsed times has been larger according to dimension. We note that the manner of comparison in GSA is as important as the $d$ threads compare two components at one time in parallel. Fig. 13(b) shows that execution of the two methods increases with the cardinality, but GSA has been always faster. The massive parallelism implemented is fruitful. In our method, all threads are functional and share the workload.

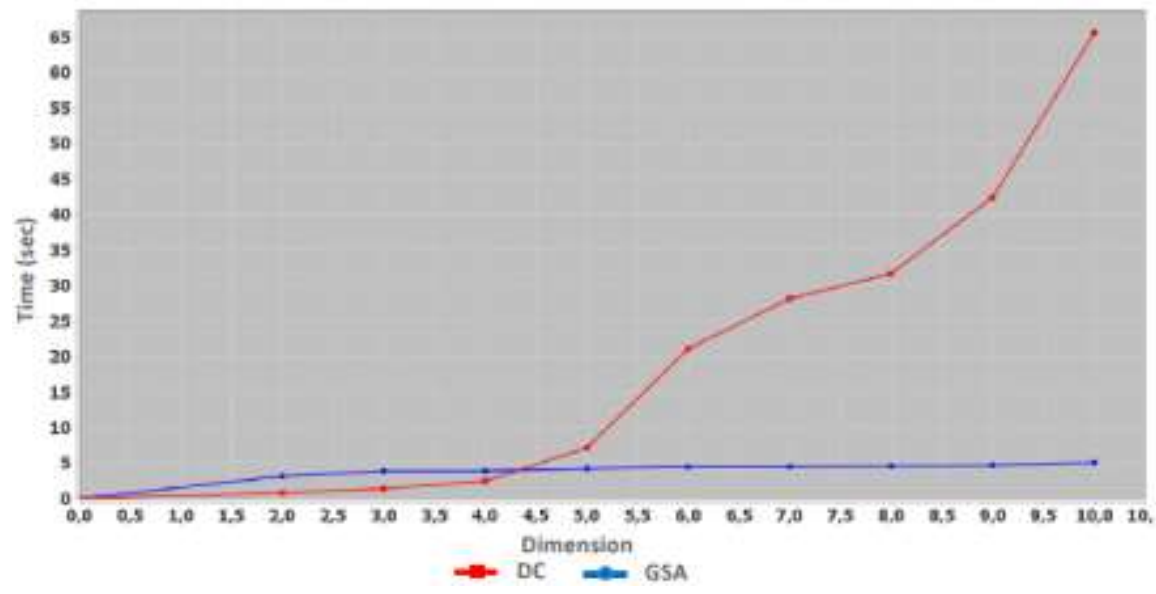

Fig. 14 (a) Elapsed time on independent data with variation of the dimensionality

Fig. 14 illustrates the comparison between DC and GSA in terms of time consumed when acting on independent data. Fig. 14(a) shows that, for small dimensions, DC is faster. The time is consumed during the computing of partitions. For every attribute of a point, DC computes at which interval it will belongs, in other words it is compared with the median. For this reason, the more the dimension increases the more the portioning time involves also.

For fewer dimensions there are fewer parts than the case for higher dimensions. In reality and in the concurrency domain, for small number of threads, sequential programs are faster than parallel ones. This is due to the synchronization which delays parallel programs. Threads must make more time for synchronization. For large dimensions and cardinalities, GSA has elapsed less time than DC. In this case, we use a higher number of threads. Consequently, more and more points are treated at every time. 


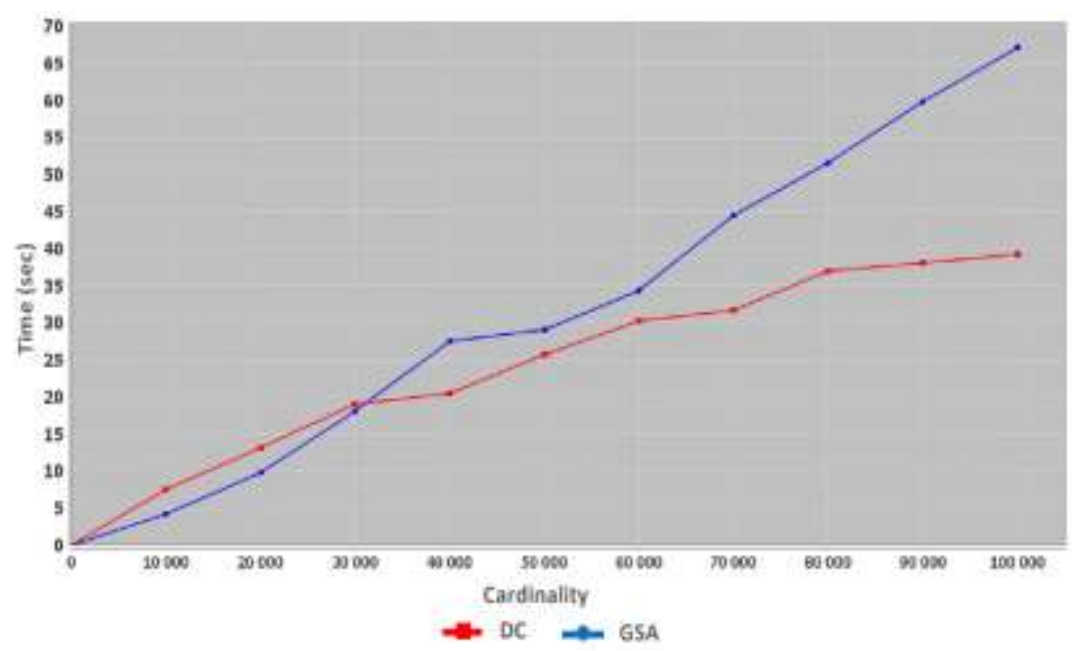

Fig. 14 (b) Elapsed time on independent data with variation of the cardinality

At the difference of precedent figure, Fig. 14(b) shows that DC has consumed fewer times than GSA. In the definition of independent data [2] a point $p$ is indifferent to be important on axis, even if it is important on one axe. For this reason, the temporary points saved in every round have been saved in the caches for more time in GSA than in DC. GSA has executed more rounds here than on correlated and anti-correlated types.

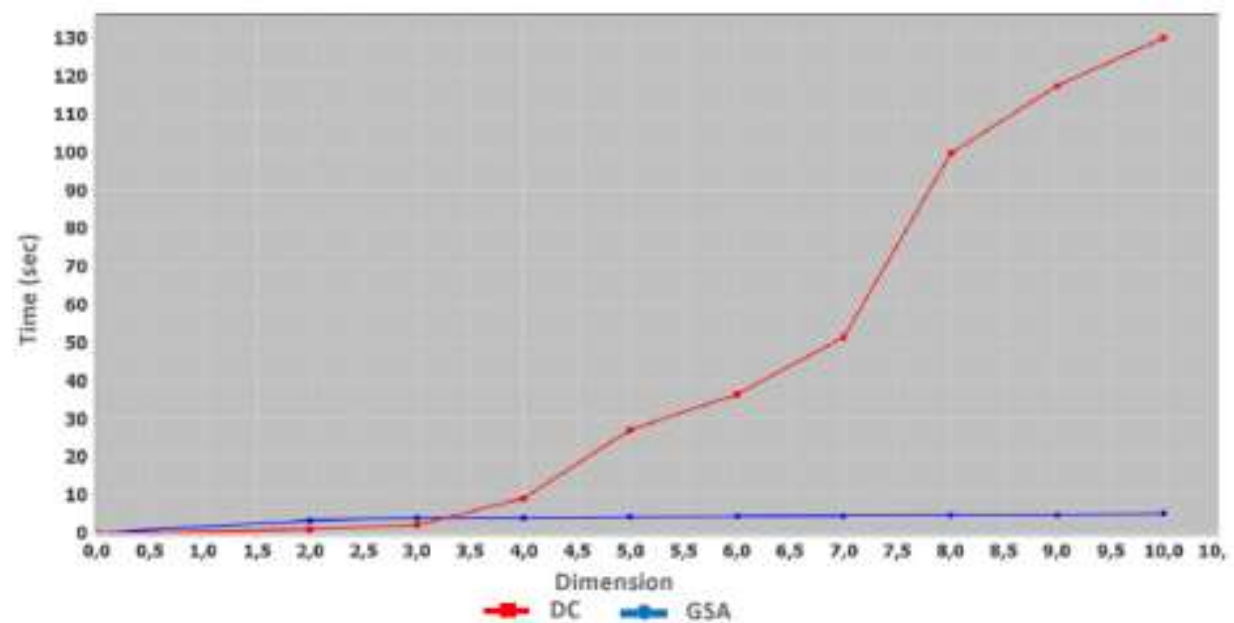

Fig.15 (a) Elapsed time on anti-correlated data with variation of dimensionality

Anti-correlated data is a difficult type of datasets to manipulate. It is characterized by a high number of skyline points. For this reason, some works have taken as research subject to find efficient methods to treat uniquely this type of datasets, for example see [9].

Fig. 15(a) shows that for small dimensions DC has taken less time than GSA, but after $d=5$ GSA starts to be faster. The time is nearly stable.

This is due to the politic of partitioning and loading data in different memories. We note that DC elapses more times as it must compute more and more parts when $d$ increases. DC computes $2^{d}$, not surely balanced, parts exactly, while all parts are balanced in the case of GSA. Fig. 15(b) shows clearly that GSA is faster as it is suitable for large datasets. We must remark that for all data types, GSA presents stable times. This is due to the SIMD model as time execution of threads is constant. 


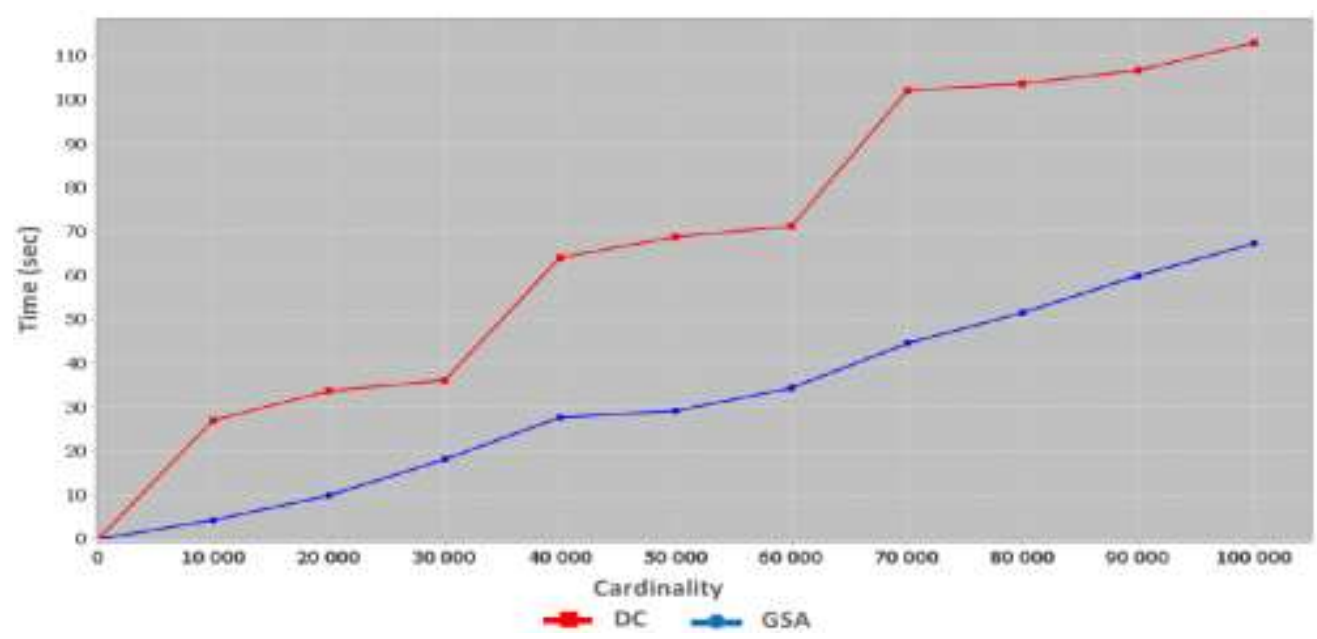

Fig. 15 (b) Elapsed time on anti-correlated data with variation of cardinality

\section{CONCLUSION}

The skyline computation is important in all fields where it is to decide about many criteria. In this paper, we have defined the skyline operator and we have given an updated state of the art. We have also presented the GPU as seen from CUDA. It is well to see that are few works which deal with skyline computation on GPU, we have presented, in detail, GNL as it is the first method in the field. The computation of the skyline is so linked to the partitioning method since the publication of the Dive-and-Conquer algorithm. In order to show the impact of the partitioning method we have presented it and detailed the generated unbalanced workload if the partitioning is not well studied. In our proposition, we partition the input according to the graphics card properties. Our portioning produces balanced parts and thus it will balance the workload.

Every BlocPart is loaded and partitioned in a well studied way for avoiding the overflow when we work with the shared memory. GSA couples the computation on both global and shared memories. For the best of our knowledge, it is the first time that such technique is proposed. In the first experimentation series, we compared GSA to GNL. These experimentations show that the elapsed time is stable and GSA has consumed less time than GNL. The most inconvenient of GNL is the non exploitation of the transitive property of the dominance relation. In the second experimentation series, and by injecting the three type datasets, we have compared GSA to DC. It results that GSA has far exceed DC especially for high dimensions and high cardinalities. In our future works, we project to extend GSA for working on multiple graphics cards. We are now hybridizing the computation on both CPU and GPU.

\section{REFERENCES}

[1] Belaicha H., Zekri L., Sekhri L. Expérimentation d'une nouvelle stratégie pour le Calcul du Skyline sur GPU, COSI 2016 volume pages 183-194, 2016

[2] Börzsonyi,S., Kossmann, D, Stocker, K. : The skyline operator. In ICDE2001. 421-430(2001).

[3] Bogh, k., Assents, I., Magnani, M. : Efficient gpu-based skyline computation. In DaMoN'13(2013).

[4] Bøgh, K.S., Chester, S., Assent, I. SkyAlign: A Portable, Work-Efficient Skyline Algorithm for Multicore and GPU Architectures. VLDB J.25(6): 817-841 (2016).

[5] Bøgh, K.S, Chester, S., Šidlauskas, D., Assent, I. Template Skycube Algorithms for Heterogeneous Parallelism on Multicore and GPU Architectures. SIGMOD'17, May 14 - 19, 2017, Raleigh, NC, USA.

[6] Choi, W., Liu, L., Boseon Yu, B. Multi-Criteria Decision Making with Skyline Computation. IEEE IRI 2012, August 8-10, 2012, Las Vegas, Nevada, USA 
[7] Chomicki, J., Godfrey, P., Gryz, J., \& Liang, D. (2005). Skyline with presorting: Theory and optimizations. In Klopotek et al. (Ed.), Intelligent Information Processing and Web Mining, Gdansk, Poland (pp. 595-604).

[8] Flynn, M.J., Some Computer Organizations and Their Effectiveness. IEEE Transactions on Computers. Vol. c-21, No.9, September 1972.

[9] Haichuan, S., Masaru, K., Skyline Operator on Anti-correlated Distributions. August 26th -30th 2013, Riva del Garda, Trento, Italy. Proceeding of the VLDB Endowment, Vol. 6, No9.

[10] Kossmann, D., Ramsak, F., Rost, S. Shooting Stars in the Sky: an Online Algorithm for Skyline Queries. VLDB, 2002

[11] Kung, H., Luccio, F., Preparata, F. On Finding the Maxima of a Set of Vectors. Journal of the ACM, 22(4), 1975.

[12] Lee, J., Kim, J., Hwang, S.: Supporting efficient distributed skyline computation using skyline views. In (DaWaK)(2009). Linz, Austria, 31 August -2 September 2009.

[13] Liknes, S., Vlachou, A., Doulkeridis, C., \& Nørvag, K. (2014). APSkyline: Improved Skyline Computation for Multicore Architectures. Proc. DASFAA (pp. 312-326).

[14] Mullesgaardy, K., Pederseny, J. L., Luy, H., \& Zhou, Y. (2014, March 24-28). Efficient Skyline Computation in MapReduce. Proc. 17th International Conference on Extending Database Technology (EDBT), Athens, Greece.

[15] Nickolls, J., Kirk, D., Graphics and Computing GPUs. Appendix_C. Computer Organization and Design. 5th edition. Morgan Kaufmann, 2014.

[16] Papadias, D., Tao, Y., Fu, G., \& Seeger, B. (2003, June 9-12). An optimal and progressive algorithm for skyline queries. ACM SIGMOD'2003, San Diego, California (pp. 467-478).

[17] Pei, J., Jin, W., Ester, M., Tao, Y.. Catching the best views of the skyline: a semantic approach based on decisive subspaces. In VLDB, pages 253-264, 2005.

[18] Reges, S., Stepp, M. Building Java Programs. A Back to Basics Approach 4th edition by. AddisonWesley. Second Edition. 2011.

[19] Tan, K., Eng, P., \& Ooi, B. (2001, September). Efficient Progressive Skyline Computation. Proceedings of the 27th International Conference on Very Large Data Bases, Rome, Italy.

[20] Vlachou, A., Doulkeridis, C., \& Kotidis, Y. (2008). Angle based Space Partitioning for Efficient Parallel Skyline Computation. Proc of ACM SIGMOD '08, Vancouver, BC, Canada.

[21] Wang, W., Zhang, J., Sun, M., Ku, W.. Efficient Parallel Spatial Skyline Evaluation Using MapReduce. Published in Proc. 20th International Conference on Extending Database Technology (EDBT), March 21-24, 2017 - Venice, Italy.

[22] Yu. B, Choi. W, Liu. L; Exploring correlation for fast skyline computation The Journal of Supercomputing Volume 73 Issue 11, November 2017 Pages 5071-5102

[23] Yuan, Y., Lin, X., Liu, Q., Wang, W., Xu, J., Yu, J., \& Zhang, Q. (2005). Efficient computation of the skyline cube. Proceedings of VLDB (pp. 241-252).

[24] Zekri L, A New Progressive Method for Computing Skyline Queries. Journal of Information Technology Research Volume 10 • Issue 3• July-September 2017.

[25] Zhang, B., Zhou, S., and Guan, J., Adapting Skyline Computation to the MapReduce Framework: Algorithms and Experiments. DASFAA Workshops 2011, LNCS 6637, pp. 403-414, 2011. 